OPEN ACCESS

Edited by:

Cosimino Malitesta,

University of Salento, Italy

Reviewed by:

Nicola Cioffi,

University of Bari Aldo Moro, Italy

Lai-Kwan Chau,

National Chung Cheng

University, Taiwan

*Correspondence:

Alia Méndez-Albores

alia.mendez@correo.buap.mx

Miguel A. González-Fuentes

miguel.gonzalezfuentes@

correo.buap.mx

Specialty section:

This article was submitted to

Analytical Chemistry,

a section of the journa

Frontiers in Chemistry

Received: 30 September 2020

Accepted: 30 November 2020

Published: 18 December 2020

Citation:

López-Castaños KA, Ortiz-Frade LA

Méndez E, Quiroga-González E,

González-Fuentes MA and Méndez-Albores A (2020) Indirect

Quantification of Glyphosate by SERS Using an Incubation Process With Hemin as the Reporter Molecule: A

Contribution to Signal Amplification

Mechanism. Front. Chem. 8:612076.

doi: 10.3389/fchem.2020.612076

\section{Indirect Quantification of Glyphosate by SERS Using an Incubation Process With Hemin as the Reporter Molecule: A Contribution to Signal Amplification Mechanism}

\author{
Karen A. López-Castaños ${ }^{1}$, Luis A. Ortiz-Frade ${ }^{2}$, Erika Méndez ${ }^{3}$, \\ Enrique Quiroga-González ${ }^{4}$, Miguel A. González-Fuentes ${ }^{3 *}$ and Alia Méndez-Albores ${ }^{1 *}$
}

${ }^{1}$ Centro de Química-ICUAP, Benemérita Universidad Autónoma de Puebla, Puebla, Mexico, ${ }^{2}$ Centro de Investigación y Desarrollo Tecnológico en Electroquímica (CIDETEQ), Pedro Escobedo, Mexico, ${ }^{3} \mathrm{Facultad}$ de Ciencias Químicas, Benemérita Universidad Autónoma de Puebla, Puebla, Mexico, ${ }^{4}$ Institute of Physics, Benemérita Universidad Autónoma de Puebla, Puebla, Mexico

The indirect determination of the most used herbicide worldwide, glyphosate, was achieved by the SERS technique using hemin chloride as the reporter molecule. An incubation process between hemin and glyphosate solutions was required to obtain a reproducible Raman signal on SERS substrates consisting of silicon decorated with Ag nanoparticles (Si-AgNPs). At $780 \mathrm{~nm}$ of excitation wavelength, SERS spectra from hemin solutions do not show extra bands in the presence of glyphosate. However, the hemin bands increase in intensity as a function of glyphosate concentration. This allows the quantification of the herbicide using as marker band the signal associated with the ring breathing mode of pyridine at $745 \mathrm{~cm}^{-1}$. The linear range was from $1 \times 10^{-10}$ to $1 \times 10^{-5} \mathrm{M}$ and the limit of detection (LOD) was $9.59 \times 10^{-12} \mathrm{M}$. This methodology was successfully applied to the quantification of the herbicide in honey. From Raman experiments with and without silver nanoparticles, it was possible to state that the hemin is the species responsible for the absorption in the absence or the presence of the herbicide via vinyl groups. Likewise, when the glyphosate concentration increases, a subtle increase occurs in the planar orientation of the vinyl group at position 2 in the porphyrin ring of hemin over the silver surface, favoring the reduction of the molecule. The total Raman signal of the hemin-glyphosate incubated solutions includes a maximized electromagnetic contribution by the use of the appropriate laser excitation, and chemical contributions related to charge transfer between silver and hemin, and from resonance properties of Raman scattering of hemin. Incubation of the reporter molecule with the analyte before the conjugation with the SERS substrate has not been explored before and could be extrapolated to other reporter-analyte systems that depend on a binding equilibrium process.

Keywords: surface enhanced Raman spectroscopy, quantification, glyphosate, reporter molecule, hemin 


\section{INTRODUCTION}

$\mathrm{N}$-phosphonomethyl glycine, known as glyphosate (GLY), is the main active ingredient of one of the most used herbicides worldwide (Avino et al., 2020). GLY presents a non-selective systemic mode of action; once it is absorbed by plants mainly through the foliage, the substance has the ability to translocate to metabolic sinks where it disables the Shikimate pathway of enzyme 5-enolpyruvylshikimate-3-phosphate synthase (EPSPS) and interrupts the synthesis of aromatic amino acids involved in the plant growth (Turner, 2020).

Since the 1970s, glyphosate has been used in an excessive and deregulate manner in agriculture. It is currently applied in field crops, including fruits, vegetables and cereals, for both conventional and genetically modified (GM). As a result, glyphosate residues are commonly found in environmental, biological, and food samples (Steinborn et al., 2016; Avino et al., 2020).

The glyphosate residue intake is a latent risk for human health since the herbicide has been cataloged as a potential carcinogen according to IARC, the International Agency for Research on Cancer from the World Health Organization (IARC, 2017). Despite the importance of glyphosate, its stability to hydrolysis and the lack of chromophore or fluorescent groups complicate its analytical determination, besides the difficulties of being separated in food matrices due to the similarity with natural amino acids.

At present, the most employed analytical technique to quantify glyphosate in food is liquid chromatography-tandem mass spectrometry (LC-MS/MS). However, this technique is expensive and requires sophisticated equipment and qualified personnel, in addition to the possibility of matrix effects (Avino et al., 2020). For this reason, research efforts are being focused on the development of alternative methods for analytical applications. In this context, Surface Enhanced Raman Spectroscopy (SERS) is among the most robust options to be employed as an alternative or pre-screen method before the use of a routine analytical technique in a wide variety of fields, including food safety (Zhao et al., 2018; Lin et al., 2020). However, this technique still presents serious drawbacks that have impeded its use as a routine analytical technique at the level of LC-MS/MS (Pérez-Jiménez et al., 2020). SERS is based on the amplification of the Raman response of an analyte when it is adsorbed on or in close proximity with the plasmon surface of metals such as $\mathrm{Au}, \mathrm{Ag}$, or $\mathrm{Cu}$, and it is capable of single-molecule identification in some cases (Demirel et al., 2018). To maximize the SERS signals, it is mandatory to combine the optimal performance of the plasmonic substrates (electromagnetic contribution, EMM) with the chemical contribution (CHEMM) of the adsorbate and from the adsorbate-substrate interaction under the effect of the incident light (Pilot et al., 2019). Examples of chemical contributions are the charge transfer between the metal and the target molecule, or vice versa, and/or non-resonant effects (static charge transfer) by the adsorption process of the molecule in its electronic ground state (Cui et al., 2010; Pilot et al., 2019). Thus, the achieved sensitivity in SERS measurements will depend on the chemical properties of the analyte and on the optimization of the Raman signal by EMM and CHEMM. So far, optimizing the SERS signal is commonly addressed through the SERS-active metal substrates (EMM). Despite the great advances in this area, the relatively high cost, low homogeneity and reproducibility of the substrates result in some of the most important drawbacks for practical applications of SERS (Mosier-Boss, 2017).

Detection of analytes by SERS has been conducted in both direct and indirect forms. The indirect form (IF) is for analytes that are not able to be adsorbed or to be close to the metal surface, resulting ideal when the target molecule possess low or null Raman vibration modes, or when the selectivity needs to be enhanced, such as in the case of oligonucleotide sequences distinction (Pyrak et al., 2019) or biomarker detection (Li et al., 2017). The IF correlates the SERS spectrum changes of a metabolite, a reaction product, or a Raman reporter molecule (RM), attached on the surface of the SERS substrate, with the concentration of the target analyte (Xu et al., 2018; Pilot et al., 2019). The use of reporter molecules is the most common way to address the indirect detection, especially in biological samples where the combination of RMs with specific antibodies also adsorbed on SERS nanoparticles (SERS tag) forms part of the detection strategy. RMs usually are small in size, present high Raman cross-sections at the selected wavelength, are photochemically stable, and show a preference for the plasmonic metal employed. They also present phenomena that contribute to the enhancement of the Raman signal, such as Raman resonance scattering properties that may result in contributions of chemical nature to the SERS signal (Li et al., 2018). Despite the wide variety of RMs reported in the literature and even of their commercial availability, the scope of their application is still underdeveloped (Sánchez-Purrà et al., 2018).

Reported methodologies to detect and quantify GLY by SERS include the direct and the indirect measurement ways. As indirect detection, we can mention de following works: Torul et al. (2010) reported an indirect detection that includes the use of gold nanorods (AuNR) derivatized with 4-mercaptophenylboronic acid as the reporter molecule. These particles were mixed with GLY in methanol, left for interaction during a specific time, and then deposited onto a silicon wafer by evaporation of the solvent for SERS measurements. Attomolar detection levels (1 $\times 10^{-16} \mathrm{mM}$ ) were achieved under this strategy, and the sensor was tested in tomato juice. Tan et al. (2017) reported a SERS strategy for the quantification of organophosphate pesticides (OPPs), including glyphosate, by using osmium carbonyl clusters on the surface of gold nanoparticles as SERS probes in a liquid medium. The analytical strategy contemplates the inhibition of thiocholine (the acetylcholinesterase catalyzed hydrolysis product of acetylthiocholine) and the subsequent decrease of thiocholine-induced aggregation of the SERS probes when OPPs are present in the sample. Changes in their aggregation modify the CO stretching vibration signal of the SERS probes at the mid-IR region $\left(1,800-2,200 \mathrm{~cm}^{-1}\right)$, making the quantification of glyphosate possible. The limit of detection was $0.1 \mathrm{ppb}$ $\left(5.91 \times 10^{-10} \mathrm{M}\right)$, and the method was evaluated in spiked beer samples. Xu et al. (2018) proposed a method based on the SERS activity of silver nanoparticles (AgNPs) in a colloidal medium through the detection of purple color dye 
(PD), a product formed during the derivatization of GLY with ninhydrin. The reported limit of detection (LOD) was $1.43 \times 10^{-8} \mathrm{M}$, and the methodology was evaluated in water samples. However, extrapolation of the methodology to foodstuff may be complicated by the well-known reaction of ninhydrin with amino acids. In the case of direct detection, De Góes et al. (2017) detected the herbicide by using Ag colloidal solutions. Detection was possible from the interaction of the negatively charged AgNPs with the respective species of GLY at different $\mathrm{pH}$ values (4-8). The LOD was $19 \mathrm{mM}$ and showed applicability in the evaluation of tap water samples in the presence of interferents, such as glufosinate-ammonium salt, AMPA, sodium chloride, and with commercial glyphosatebased herbicides.

Hemin is the hemoglobin oxidation product; it is a protoporphyrin IX containing an iron (III) center (Heme B) with a chloride ligand. As a biosensor element, it has been used for electrochemical detection of 2,4,6 trichlorophenol (Zhang et al., 2018), ascorbic acid, dopamine, uric acid (Zou et al., 2015), and glucose (He et al., 2016). Likewise, the hemin/Gquadruplex Dnazyme, an artificial enzyme with peroxidase activity, has been employed to develop electrochemical and colorimetric detection of a variety of chemical and biological targets (Alizadeh et al., 2017). The use of hemin as a reporter molecule for the optical determination of analytes by Raman has not been explored; however, it presents multiple advantages such as a variety of electronic transitions in the visible and near-infrared regions and its well-characterized Raman spectra at different excitation wavelengths (Franzen et al., 2002). Therefore, in this work, hemin chloride was used as a Raman reporter molecule to determine glyphosate. Also, in order to potentiate the enhancement and stability of the Raman signal, the use of an incubation process between the Raman reporter and the analyte, previously to SERS measurements, was tested for the first time. The SERS experiments were conducted on Si-AgNPs substrates using the wet mode of spectrum acquisition developed in our research group ("the drop technique"), which allows the in situ evaluation of the adsorption process on a simple and easy way.

\section{MATERIALS AND METHODS}

\section{Chemicals and Materials}

$\mathrm{AgNO}_{3}$ 99\%, hydrofluoric acid (HF, 48\%), sodium hydroxide $(\mathrm{NaOH}, 97 \%)$, sodium borate decahydrate $\left(\mathrm{Na}_{2} \mathrm{~B}_{4} \mathrm{O}_{7} \cdot 10 \mathrm{H}_{2} \mathrm{O}\right)$, hemin $\left(\mathrm{C}_{34} \mathrm{H}_{32} \mathrm{ClFeN}_{4} \mathrm{O}_{4}, \mathrm{FW} 651.96 \mathrm{~g} / \mathrm{mol}\right)$, were purchased from Sigma-Aldrich. Glyphosate (99.5\%) was procured from Chem Service. All reagents were used without further purification. Silicon wafers (Si) type $\mathrm{P}$, doped with boron (B), orientation (100), and resistivity of $15-25(\Omega \mathrm{cm})$ were purchased from Siltronic AG. Deionized water with a resistivity of $\sim 18 \mathrm{M} \Omega \mathrm{cm}$ was obtained by using a Millipore MilliQ Plus water purification system. Acetone $\left(\mathrm{C}_{3} \mathrm{H}_{6} \mathrm{O}, 98 \%\right)$ was obtained from CTR Scientific, and nitrogen gas (99.9\%) was purchased from Infra.

\section{Preparation and Characterization of Si-AgNPs Substrates}

The Si-AgNPs substrates were synthesized by chemical deposition. Pieces of $1 \mathrm{~cm}^{2}$ of polished p-type (100) Si wafers with a resistivity range of $15-25 \Omega \mathrm{cm}$ (electronic grade) were used as substrates. Si pieces were subjected to exhaustive washing with water for $10 \mathrm{~min}$ and acetone for $5 \mathrm{~min}$. After that, the pieces were rinsed with water. Then, Si pieces were immersed for $7 \mathrm{~min}$ into the deposition solution prepared with $0.2 \mathrm{~mL}$ of $48 \% \mathrm{HF}$ and $10 \mathrm{ml}$ of $\mathrm{AgNO}_{3} 5.88 \times 10^{-4} \mathrm{M}$. HF reacts with $\mathrm{Si}$ producing soluble $\mathrm{H}_{2} \mathrm{SiF}_{6}$ and electrons. The electrons reduce the $\mathrm{Ag}^{+}$ions of the plating solution, producing elemental $\mathrm{Ag}$ particles on the surface of Si. A thorough explanation of the reaction mechanism is given in Aca-López et al. (2020). After deposition, the Si pieces were immediately immersed in distilled water to stop the deposition process. To prevent oxidation of the deposits, every sample was dried with a flux of nitrogen.

For the optical characterization of the Si-AgNPs, UV-Vis diffuse reflectance measurements were performed using a $\mathrm{Si}$ substrate spectrum as a reference. The measurements were carried out using a Cary $50 \mathrm{UV}$-Vis spectrophotometer from Varian Instruments, equipped with an integrating sphere. A homemade sample holder made of Teflon was used to fix the samples during the measurements. UV-Vis diffuse reflectance spectra were corrected by subtracting the spectrum of the Teflon holder. All spectra were normalized to the maximum. 1-R spectra are reported for all samples.

The morphological characterization, AFM images were obtained using a SmartSPM ${ }^{\mathrm{TM}} 1000$ atomic force microscope (Horiba Scientific) with the "Top mode" tool. Silicon cantilevers (AppNano) of $52 \mu \mathrm{m}$ width, $0.8-8.9 \mathrm{~N} \mathrm{~m}^{-1}$ spring constant, and $36-98 \mathrm{kHz}$ resonance frequency in the air were used. Scan speed of $1.0 \mathrm{~Hz}$ and $450 \times 450$ pixels per line resolution were employed. Images were processed using the Gwydion 2.30 software.

\section{Sample Preparation}

Solutions of hemin were prepared fresh by dissolving hemin chloride to a concentration of $25 \mu \mathrm{M}$ in $0.25 \mathrm{M}$ borax ( $\mathrm{pH}$ 9.26). Glyphosate solutions $\left(1 \times 10^{-4}, 1 \times 10^{-3}, 1 \times 10^{-2}, 0.1,1\right.$ and $10 \mu \mathrm{M}$ ) were prepared by dilution of a $0.01 \mathrm{M}$ glyphosate stock solution. The sample containing glyphosate (deionized water or spiked honey solutions) was incubated with a hemin-borate solution in a volume ratio of $1: 1$ and then left to rest for $48 \mathrm{~h}$ at $-4^{\circ} \mathrm{C}$ without light exposition. UV-Vis studies of hemin and hemin-glyphosate solutions were carried out using a Cary 50 UVVis spectrophotometer from a Varian instrument. With the same technique, hemin concentration was corroborated by measuring the absorbance of the solution at $\lambda=385 \mathrm{~nm}\left(€=5.84 \times 10^{4}\right.$ $\left.\mathrm{cm}^{-1} \mathrm{M}^{-1}\right)$.

\section{Preparation of Real Spiked Samples}

A series of honey samples artificially contaminated with glyphosate at concentrations of $0.1 \mathrm{nM}, 1 \mathrm{nM}, 10 \mathrm{nM}, 100 \mathrm{nM}$, $1 \mu \mathrm{M}$, and $10 \mu \mathrm{M}$ were prepared as follows: $1 \mathrm{~g}$ of honey was weighed into a $5-\mathrm{mL}$ volumetric flask and filled with a solution of $0.25 \mathrm{M}$ borate. The sample was diluted with the necessary 
volume of borate solution to allow the honey dissolution; after that, $25 \mu \mathrm{M}$ of a solution with the desired concentration of glyphosate was added to the mixture, and the volume completed to $5 \mathrm{~mL}$ with a borate solution. It was necessary that this solution was adjusted to $\mathrm{pH} 9.2$ with $1 \mathrm{M} \mathrm{NaOH}$ due to the acidity of the honey.

\section{Raman Spectroscopy Measurements}

Raman spectra were collected using a Micro-Raman system (Xplora Plus microscope from Horiba) equipped with a confocal microscope (Olympus BX51). Lasers of 532 and $780 \mathrm{~nm}$ wavelength excitation were employed at 20 and $100 \mathrm{~mW}$ power, respectively. The instrument was calibrated using the 520.71 $\mathrm{cm}^{-1}$ band of a silicon wafer. All spectra were obtained in aqueous media using the "drop technique." A 10x objective was used. The acquisition parameters of the spectra were set to 1,200

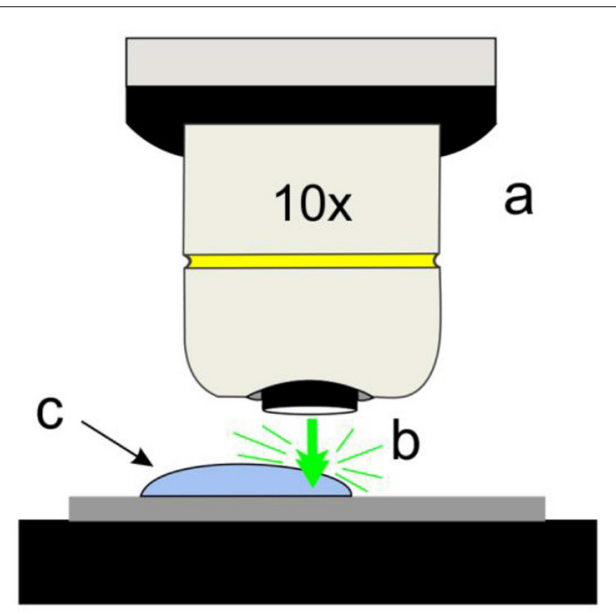

FIGURE 1 | Simplified scheme of the drop technique: (a) objective, (b) laser beam, (c) drop of sample on Si-Ag NPs substrates. $\mathrm{g} \cdot \mathrm{mm}^{-1}$ grating, slit $200(\mu \mathrm{m})$, hole $300(\mu \mathrm{m}), 12 \mathrm{~s}$ of acquisition time, and an accumulation of five spectra. The control of the equipment for data acquisition and preliminary analysis was carried out with LabSpec6 software.

\section{The "Drop Technique"}

SERS measurements were carried out using the "drop technique," which consists of the following steps: (1) the Raman microscope is set to focalize the surface of the SERS substrate with the aid of a CCD camera; (2) a drop of the analyte is deposited on the surface of the SERS substrate by using a micropipette; (3) the measurement is performed on the droplet at $10 \mu \mathrm{m}$ from its boundary. The acquisition parameters are selected in such a way to avoid the evaporation of the droplet during the measurement. The Raman experimental setup is depicted in Figure $\mathbf{1 .}$

Through the use of this new drop technique, it is possible to carry out Raman experiments on SERS substrates with liquid media in a simple form, in comparison with other strategies reported in the literature, such as microfluidic system (LoCSERS) (März et al., 2011) or on a drop of the liquid sample placed on the SERS substrate, which is covered with a thin coverslip (Peters et al., 2015).

\section{RESULTS AND DISCUSSION}

\section{Characterization of Si-AgNPs Substrates}

The maximum electromagnetic contribution to the SERS intensity can be obtained from the Si-AgNPs substrates and corresponds to the surface plasmon resonance (LSPR) of the silver nanoparticles. AFM and diffuse reflectance UV-Vis spectroscopy were used in order to correlate the topographic characteristics of the silver deposit on silicon with the plasmonic response of the $\mathrm{Si}$-AgNPs substrates. Figure $2 \mathrm{~A}$ shows a typical AFM image of the Si-AgNPs substrate used for this study. It can be observed that Ag nanoparticles have a dispersion of sizes; however, most of the particles tend to be semi-spherical.
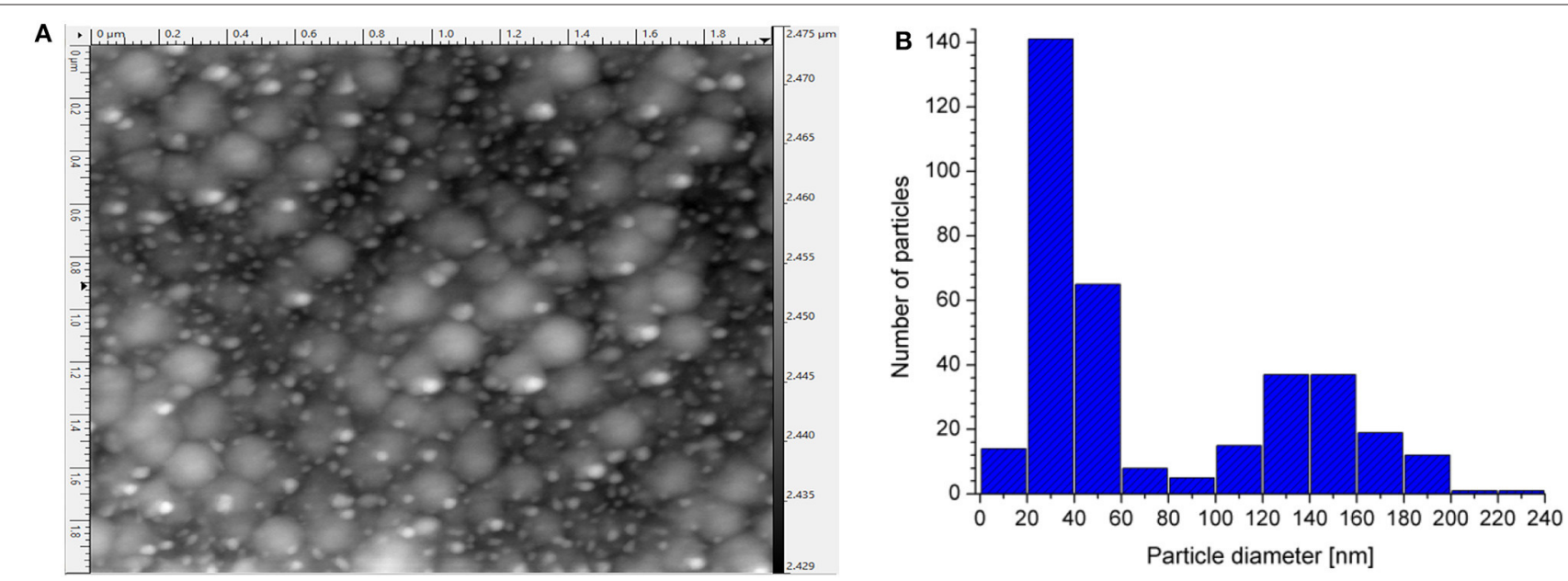

FIGURE 2 | (A) Typical AFM micrograph of the Si-AgNPs substrates. (B) Histogram of the set of Ag nanoparticles from 2 A micrograph. 


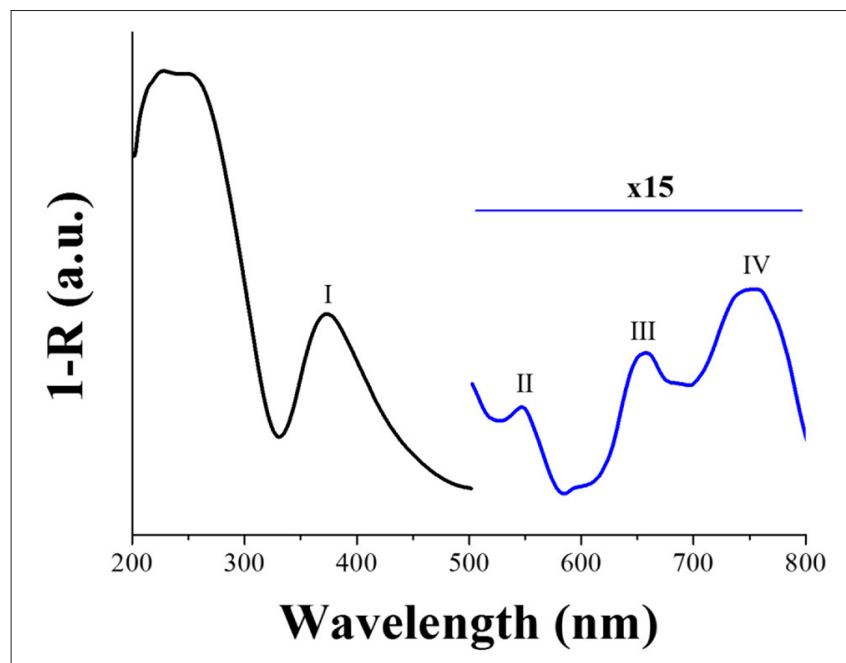

FIGURE 3 | Graph of 1-R vs. wavelength. Absorption bands indicated as I, II, III, and IV, respectively.

The corresponding histogram of the size distribution of the Ag particles is presented in Figure 2B.

One can identify two main groups of particle sizes. One group has an average size of $140 \mathrm{~nm}$, and about $60 \%$ of the particles of this group have sizes between 121 and $160 \mathrm{~nm}$. The other group has sizes below $60 \mathrm{~nm}$, where $90 \%$ of the particles have sizes between 21 and $60 \mathrm{~nm}$. A mixture of particles of different sizes can be observed through the sample. In most of the reports of SERS substrates, the authors try to obtain monodisperse particles (Lee et al., 2019; Chen et al., 2020), but the techniques are more complicated than ours. The great advantage of the substrates of the present work is their simplicity and low fabrication cost.

Dispersion of size in plasmonic particles produces a broad plasmonic spectrum that could be useful for performing SERS at different wavelengths (Mao et al., 2020). A similar effect could be also obtained with dendrites (Lu et al., 2020) or particles with a dispersion of forms (Yin et al., 2020).

The plasmonic response of the $\mathrm{Si}$-AgNPs substrates was evaluated by diffuse reflectance UV-Vis spectroscopy. Figure 3 shows a graph of $1-\mathrm{R}$ vs. $\lambda$. Four Localized Surface Plasmon Resonances (LSPRs) were identified: band I (371 nm), band II $(543 \mathrm{~nm})$, band III $(657 \mathrm{~nm})$, and band IV $(752 \mathrm{~nm})$. Similar multi-LSPRs were reported by Kosović et al. (2015) for silver nanoparticles exhibiting comparable morphological features to the Si-AgNPs substrates. The width of the most intense LSPR band (band I) is the result of the broad size distribution of particles, while the appearance of the rest of the bands (II, III, and IV) is most likely due to the different aspect ratios of the particles and their coalescence (Sharma et al., 2020).

Spherical particles exhibit one resonance band due to the presence of a single dipole; in the case of particles of $40 \mathrm{~nm}$ size, the band is intense and centered at about $400 \mathrm{~nm}$ (Lee et al., 2008). However, for quasi-spherical particles with an aspect ratio larger than 1, a double resonance occurs: the intense SPR band blue shift and an extra band appears at wavelengths above $500 \mathrm{~nm}$
(Amirjani and Haghshenas, 2018). On the other hand, an increase of the aspect ratio or the overall size of the particle provokes a red-shift of this additional peak (Sharma et al., 2020).

Thus, the origin of the bands at $371 \mathrm{~nm}$ and $543 \mathrm{~nm}$ in Figure 3 could be arising from semi-spherical particles with sizes below $60 \mathrm{~nm}$. Likewise, bands at 371 and $657 \mathrm{~nm}$ could be originated from larger particles, as those with an average size of $140 \mathrm{~nm}$, as reported by Kosović et al. (2015). Therefore, the band at $371 \mathrm{~nm}$ may result from the contribution of both particle sizes, which explains its broadness.

The band at $752 \mathrm{~nm}$ is due to the coalescence of the larger particles. As it can be observed in the micrograph (Figure 2A), groups of particles are found. It is well-known that the coalescence of particles generates multipole oscillations and new plasmonic modes at higher wavelengths (Amirjani and Haghshenas, 2018). Additionally, low-intensity shoulders were also observed at about 600 and $680 \mathrm{~nm}$. These additional modes may also be related to the aforementioned Ag aggregated particles, specifically to their interaction. It is reported that a redshift as large as $70 \mathrm{~nm}$ in the Raman peak position may occur if particles are close together (when the ratio of the gap over the particle diameter is smaller than 0.1). A similar shift occurs when contiguous particles differ in size (Drozdowicz-Tomsia and Goldys, 2011).

\section{UV-Vis Spectroscopy of Hemin-Glyphosate Incubated Solutions}

It has been previously reported that the UV-Vis spectra of hemin dissolved in borate solution show multiple electronic transitions in the visible and near-infrared wavelength regions (Wood et al., 2004). However, the spectral behavior of aqueous solutions of hemin changes as a function of time (Maehly and Akeson, 1958). Considering the above information, in this work the incubation of a mixture of solutions of glyphosate (at different concentrations, from $1 \times 10^{-4}$ to $\left.10 \mu \mathrm{M}\right)$ and hemin $(25 \mu \mathrm{M})$ in a volume ratio of 1:1 was carried out for $48 \mathrm{~h}$ in order to allow the binding equilibrium to occur during the incubation exposition process and before SERS experiments.

The characterization by UV-Vis spectroscopy of the heminglyphosate $(1 \mu \mathrm{M})$ mixture before and after incubation is shown in Figure 4. For comparison, the absorption spectra of $25 \mu \mathrm{M}$ hemin solution in borate, freshly prepared and $48 \mathrm{~h}$ aged are added.

All spectra present the same pattern as the freshly prepared hemin-borate solution: the characteristic partitioned Soret band $\mathrm{B}$ of the formation of hemin dimmers is present with a maximum intensity at $383 \mathrm{~nm}$ (Aratani et al., 2002). In the range from 450 to $900 \mathrm{~nm}$, several bands are observed [I $(493 \mathrm{~nm})$, II $(531 \mathrm{~nm})$, III $(610 \mathrm{~nm})$, IV $(695 \mathrm{~nm}), \mathrm{V}(817 \mathrm{~nm})]$. Bands I and II, known as $\mathrm{Q}$ bands, are assigned to vibronic components of porphyrin ring $\pi \rightarrow \pi^{*}$ transition modulated by the Fe(III) ion (Toader et al., 2013). Bands III, IV, and V are associated with charge transfer (CT) from $d \pi$ orbitals in the iron atom to the porphyrin ring with a variable contribution of the $\pi-\pi^{*}$ transition (Arbelo-López et al., 2018). However, bands IV and V are absent when hemin concentration is $<15 \mu \mathrm{M}$ (see Supplementary Figure $\mathbf{1}$ ) as it has 


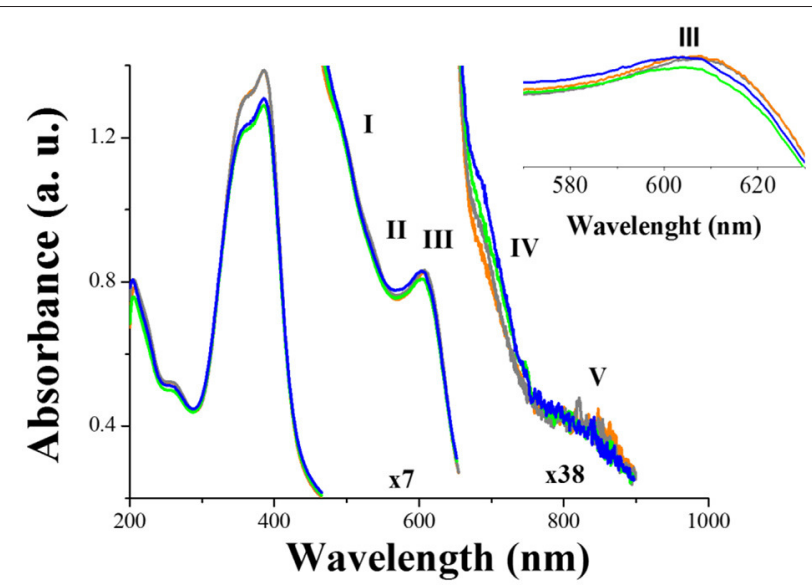

FIGURE 4 | Absorbance spectra of: fresh solution of $25 \mu \mathrm{M}$ hemin in $0.25 \mathrm{M}$ borate solution (-); fresh mixture of hemin-borate and $1 \mu \mathrm{M}$ glyphosate in borate (-); $25 \mu \mathrm{M}$ of hemin dissolved in $0.25 \mathrm{M}$ borate solution and after $48 \mathrm{~h}$ of incubation (-); mixture of hemin-borate and $1 \mu \mathrm{M}$ glyphosate in borate after $48 \mathrm{~h}$ of incubation ( - ). The incubation was made at $-4^{\circ} \mathrm{C}$ without light exposition. Inset: Amplification in the $550-630 \mathrm{~nm}$ range.

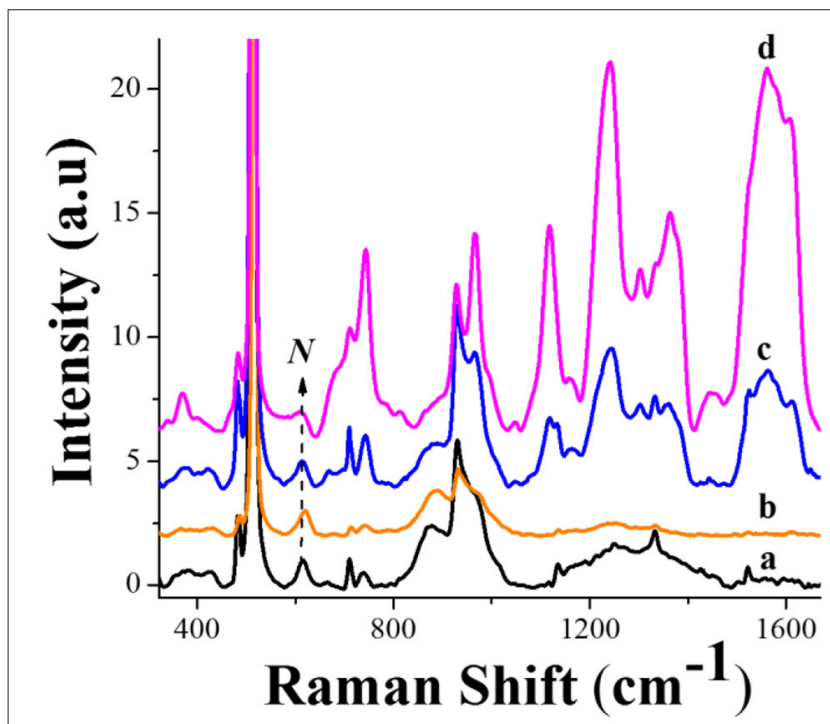

FIGURE 5 | SERS spectra of the systems: $0.25 \mathrm{M}$ borate solution (curve a), 1 $\mu \mathrm{M}$ GLY in $0.25 \mathrm{M}$ borate solution (curve b), $25 \mu \mathrm{M}$ of hemin in $0.25 \mathrm{M}$ borate solution (curve c), and a mixture of the solutions of $25 \mu \mathrm{M}$ of hemin in borate with $1 \mu \mathrm{M}$ of glyphosate in water (curve d) after $48 \mathrm{~h}$ incubation.

been already reported (Nath et al., 2017). Thus, the presence of these bands indicates the molecular aggregation of hemin (Wood et al., 2004), and therefore the presence of excitonic interactions (Webster et al., 2009).

After aging, the spectral change of the hemin-glyphosate mixture and the hemin-borate solution is practically the same: The Soret band undergoes hypochromicity of about 3.5\%, with no change in its adsorption position and bandwidth, which indicates the dissociation of hemin dimers over time. The $\mathrm{Q}$ bands do not show any modification; therefore, there is no change in the local environment of the hemin aggregates, even in the presence of glyphosate (Liu et al., 2018). Bands III and IV of hemin in the hemin-glyphosate spectra are slightly affected by the incubation process. Both bands undergo a slight hypochromic shift (blue) of about $3 \mathrm{~nm}$ (broken curves), indicating that the binding step for reaching equilibrium involves a subtle increase in the overlap between $\pi$ porphyrin and metal $d \pi$ ( $d x z$ or $d$ $\mathrm{dxz}$ ) orbitals without an influence in the $\pi$ to $\pi^{*}$ energy gap in porphyrin electronic spectra (Aarabi et al., 2019).

The analysis of UV spectra in Figure $\mathbf{4}$ does not show evidence of a strong interaction between glyphosate and hemin. However, since experiments were performed at a $\mathrm{pH}$ of 9.2, hemin exists predominantly in the form of dimmers, with the axial $\mathrm{OH}-$ ligands pointing outwards (Scolaro et al., 2002). Meanwhile, the dominant species of glyphosate is $\mathrm{HL}^{2-}$, with its two molecular ends negatively charged by the deprotonation of one oxygen at the carboxylate and phosphonate groups (Ehrl et al., 2018; Lopes Catão and López-Castillo, 2018). Therefore, glyphosate would be expected to interact with $\mathrm{OH}$ ions by hydrogen bonds.

\section{SERS Spectra of the Hemin-Glyphosate Mixture After the Incubation Process}

When the laser used in SERS experiments corresponds to the excitation of LSPR of metal nanoparticles with a simultaneous electronic absorption of the molecule adsorbed on the substrate, an enhancement of the Raman signal additional to that corresponding to the electromagnetic contribution arises. This phenomenon is known as Resonance Raman Scattering (RRS) (Murgida and Hildebrandt, 2001). At $780 \mathrm{~nm}$, the onset of the electronic transition assigned as band $\mathrm{V}$ is observed in hemin and the mixture of hemin-glyphosate solutions after incubation process (Figure 4): the signal is a charge transfer (CT) transition and involves promotions between $\pi$ porphyrin and metal $d \pi$ (dxz or d dxz) orbitals (Wood et al., 2004). At this wavelength value, the Si-AgNPs substrates exhibit a LSRP signal (band IV) (Figure 3). Thus, the SERS response of both solutions (hemin and hemin-glyphosate) must be composed of contributions of the electromagnetic and electronic structure of chemical species due to the resonance enhancement effect.

Figure 5 shows the SERS spectra of the systems: $0.25 \mathrm{M}$ borate solution (curve a), $1 \mu \mathrm{M}$ GLY in $0.25 \mathrm{M}$ borate solution (curve b), $25 \mu \mathrm{M}$ of hemin in $0.25 \mathrm{M}$ borate solution (curve c), and the hemin-glyphosate mixture containing $1 \mu \mathrm{M}$ of glyphosate (curve d) after incubation. The most intense band at $515 \mathrm{~cm}^{-1}$ corresponds to the silicon wafer.

The SERS spectrum of borate solution (Figure 5, curve a) presents seven defined bands at 511, 613, 745, 930, 1,136, 1,333, and $1,524 \mathrm{~cm}^{-1}$. All signals except for the one at $745 \mathrm{~cm}^{-1}$ come from the silicon wafer (Supplementary Figure 2), while the band at $745 \mathrm{~cm}^{-1}$ appears in the presence of the borate medium and can be assigned to the vibrations of the tetrahydroxy borate ion $\left(\mathrm{B}(\mathrm{OH})_{4}^{-}\right)$near the Si-AgNPs substrate (Yongquan et al., 2013). However, bands at $613 \mathrm{~cm}^{-1}$ and $930 \mathrm{~cm}^{-1}$ can also possess the contributions from $\mathrm{B}_{3} \mathrm{O}_{3}(\mathrm{OH})_{4}^{-}$ions and from the $\mathrm{BO}_{3}^{3-}$ trigonal 
unit of the $\mathrm{Na}_{2} \mathrm{~B}_{4} \mathrm{O}_{7}$ molecule (Norrel et al., 2003; Yongquan et al., 2013).

Curve b of Figure 5 corresponds to the borate-glyphosate system, and the spectrum presents the same behavior as in pure borate solution, with a decrease in the total Raman intensity. The lack of glyphosate signals is consistent with the behavior obtained by the water-glyphosate system (Supplementary Figure 2) at the same herbicide concentration. The low affinity of glyphosate for

TABLE 1 | Band assignments, symmetry term, and local coordinates from SERS spectra for hemin in borate after incubation during $48 \mathrm{~h}$.

\begin{tabular}{|c|c|c|c|}
\hline $\begin{array}{l}\text { Wavenumber } \\
\left(\mathrm{cm}^{-1}\right)\end{array}$ & Assignment & Symmetry & $\begin{array}{l}\text { Term local } \\
\text { coordinate }\end{array}$ \\
\hline 341 & v8 & $v(\mathrm{Fe}-\mathrm{N})$ & A1g \\
\hline 372 & v35 & $\delta(\mathrm{C} \beta \mathrm{CcCd})_{6,7 \text { propionate }}$ & A1g \\
\hline 407 & & $\delta(\mathrm{C} \beta \mathrm{CaCb})_{4-\text { vinyl }}$ & \\
\hline 426 & & $\delta(\mathrm{C} \beta \mathrm{CaCb})_{2-\text { vinyl }}$ & \\
\hline 741 & $v 15$ & $v$ (pyr breathing)non-totally sym & $\mathrm{B}_{1 \mathrm{~g}}$ \\
\hline 932 & $v 46$ & $\delta$ (pyr deform)asym & $E_{u}$ \\
\hline 968 & $v 32+v 35$ & $\delta$ (porph def) $+\delta$ (pyr transl) & A1g \\
\hline 1,120 & & $\nu(\mathrm{Cb}-\mathrm{C} \alpha($ vinyl $)+\delta(\mathrm{Cb}-\mathrm{R})$ * & \\
\hline 1,246 & $v 13$ & $\delta\left(\mathrm{C}_{\mathrm{m}} \mathrm{H}\right)$ non-totally sym & B1g \\
\hline 1,301 & & $\delta(\mathrm{CaH}=)$ & \\
\hline 1,366 & $v 4$ & $v(\mathrm{Ca}=\mathrm{N})$ sym & A1g \\
\hline 1,442 & & $\delta(=\mathrm{CbH} 2)$ sym & \\
\hline 1,565 & v2 & $v\left(\mathrm{C}_{\mathrm{b}} \mathrm{C}_{\mathrm{b}}\right) \mathrm{sym}$ & A1g \\
\hline 1,612 & & $v\left(\mathrm{Ca}=\mathrm{C}_{\mathrm{b}}\right)$ & \\
\hline
\end{tabular}

Excitation wavelength: $780 \mathrm{~nm}$.

$v=$ in-plane stretch.

$\delta=$ deformation mode.

Based on the works of Wood et al. (2011), Hu et al. (1996), and Wood et al. (2004).

*Symmetry based from Desbois et al. (1984). the Ag surface at the herbicide concentrations used in this work was reported recently by Feis et al. (2020).

The SERS spectrum of hemin-borate solutions after $48 \mathrm{~h}$ of their preparation (Figure 5, curve c), adds bands at 967, $1,121,1,244,1,303,1,366$, and $1,558 \mathrm{~cm}^{-1}$, and provokes the enhancement of the 930 and $745 \mathrm{~cm}^{-1}$ bands, suggesting the co-adsorption of $\mathrm{B}(\mathrm{OH})_{4}^{-}$with hemin.

When glyphosate solution is incubated with the hemin solution (curve d), the SERS spectra do not show any new bands associated with the herbicide; however, the hemin peaks show an increase in intensity. Table 1 summarizes the assignments of the hemin bands where modes are designed considering the $\mathrm{D}_{4 \mathrm{~h}}$ point group symmetry.

\section{Origin of the SERS Effect}

The SERS spectra of hemin-glyphosate incubation mixtures containing different concentrations of GLY are reported in Figure 6A. All bands of hemin grow as a function of the glyphosate concentration in solution. It is important to highlight the importance of the incubation time to obtain reproducible Raman signal intensity in the assays since, without $48 \mathrm{~h}$ of incubation, the acquisition of a distinguishable trend in the Raman intensity is not possible (see Supplementary Figure 3 in supporting information). In order to understand the origin of the SERS effect that allows the continuous increase of practically all the bands of hemin as a function of glyphosate concentration in the incubated solutions, an in-depth analysis of the SERS experiments was conducted.

From Figure 6A and Table 1, the Eu infrared active mode is indicative of the asymmetry in the vinyl substituents of hemin on the silver nanoparticles (Choi et al., 1982). Moreover, the domain of modes attributable to the vinyl groups $\left[\delta(\mathrm{C} \beta \mathrm{CaCb})_{4-\text { vinyl }}, \delta(\mathrm{C} \beta \mathrm{CaCb})_{2 \text {-vinyl }}, \nu(\mathrm{Cb}-\mathrm{C} \alpha(\right.$ vinyl $)+\delta(\mathrm{Cb}-$ $\left.\mathrm{R}), \quad \delta(\mathrm{CaH}=), \delta\left(=\mathrm{CbH}_{2}\right), \quad v(\mathrm{Ca}=\mathrm{Cb})\right]$, implies that hemin is adsorbed on silver through their vinyl substituents in the
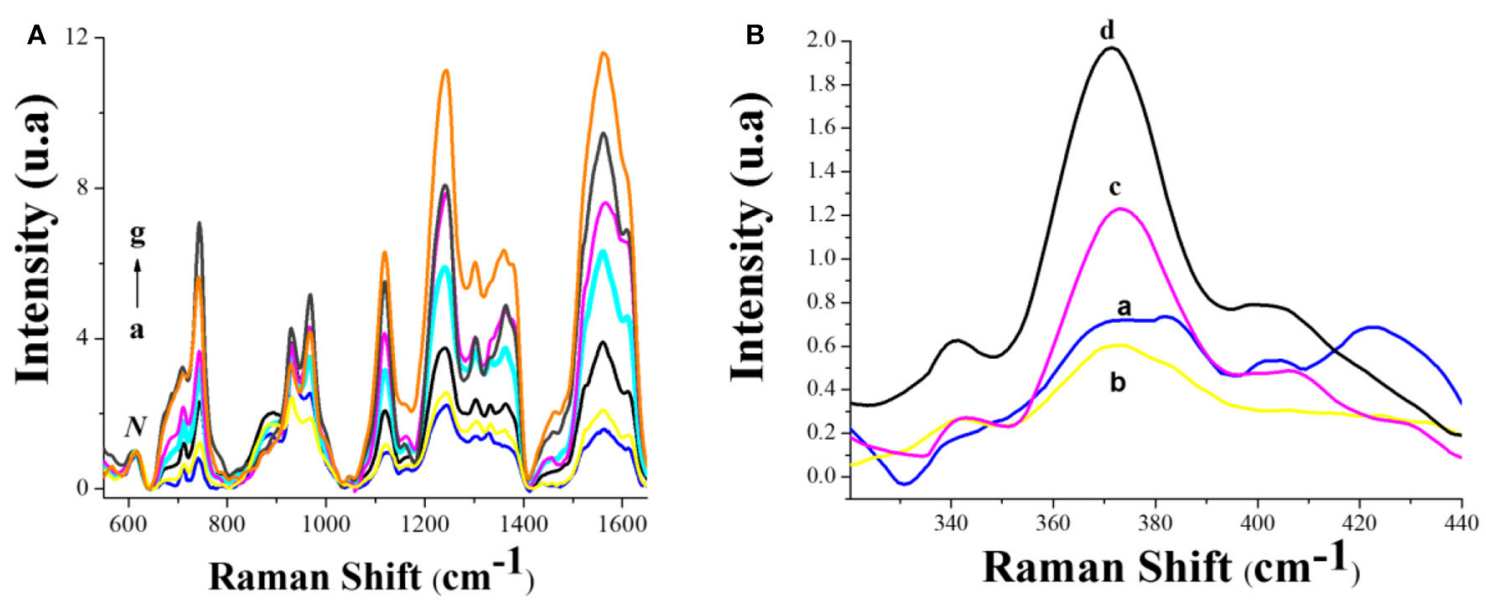

FIGURE 6 | (A) SERS spectra from 540 to $1,680 \mathrm{~cm}^{-1}$ of hemin-glyphosate incubation mixtures containing different concentrations of GLY: (a) 0, (b) $0.1 \mathrm{nM}$, (c) $1 \mathrm{nM}$, (d) $10 \mathrm{nM},(\mathrm{e}) 100 \mathrm{nM}$, (f) $1 \mu \mathrm{M}$, (g) $10 \mu \mathrm{M}$. (B) SERS spectra from 500 to $1,700 \mathrm{~cm}^{-1}$ of the incubated mixtures of hemin-glyphosate solutions containing different concentrations of GLY: (a) 0, (b) $0.1 \mathrm{nM}$, (c) $100 \mathrm{nM}$, (d) $1 \mu \mathrm{M}$. N: band used for normalization. 
presence and absence of glyphosate. This can be confirmed with the Raman spectra of the same samples, on the silicon wafer without silver nanoparticles (resonance Raman (rR) experiments), where only a single band of vinyl at $1,615 \mathrm{~cm}^{-1}$ appears (Supplementary Figure 4 of supporting information).

The band at around $1,614 \mathrm{~cm}^{-1}$ contains contributions from the two vinyl side chains of hemin that converge at the same wavenumber value due to the coplanar orientation of both groups with the porphyrin plane (Milazzo et al., 2020). On SERS substrates (see Figure 6A), the wavenumber value of the 1,614 $\mathrm{cm}^{-1}$ band undergoes a gradual down-shift when glyphosate concentration is increased in the hemin solution, reaching a value of $1,609 \mathrm{~cm}^{-1}$ at the highest tested concentration $\left(1 \times 10^{-5}\right.$ $\mathrm{M})$. However, in the rR spectrum of hemin, the frequency value of this mode remains unchanged in the presence of glyphosate (Supplementary Figure 4). This result suggests a gradual change in the planar orientation of the vinyl groups of hemin due to its binding onto the silver substrate, presenting a major conjugation between the vinyl substituent and the porphyrin macrocycle at the higher concentration of glyphosate (higher degree of trans configuration) (Marzocchi and Smulevich, 2003). Although the resonance response to an increased conjugation should be accompanied by a decrease in the intensity and a down-shift of the wavelength value of the $\nu 2$ mode (Rwere et al., 2008), the presence of glyphosate makes this mode to show an increase in intensity without a change in position due to an increase in the proximity of the vinyl groups to the surface of silver (electromagnetic enhanced mechanism).

The evaluation of the high wavenumber range in Figure 6A includes a resurgence of the band $v 4$, which becomes wellresolved at the highest glyphosate concentration $\left(1 \times 10^{-5} \mathrm{M}\right)$. The $v 4$ mode is associated with the $\mathrm{C}-\mathrm{N}$ stretch vibrations of the pyrrole subunits and is considered the oxidation-state marker band. The occurrence of $v 4$ mode at $1,366 \mathrm{~cm}^{-1}$ is consistent with the Fe atom in its Fe(II) oxidation state (Wood et al., 2001). The position of $v 15$ at $744 \mathrm{~cm}^{-1}$ corroborates the presence of reduced hemin on the surface of the Si-AgNPs substrates (Zheng et al., 2003).

At low wavenumber values (Figure 6B), the presence of glyphosate provokes the attenuation of intensity in the band at $421 \mathrm{~cm}^{-1}$ assigned to the out-of-plane bending motion of the vinyl group attached to the pyrrole II group (Rwere et al., 2008). This phenomenon is associated with an increase in its degree of planarity over the surface. As shown in the inset of Supplementary Figure 4, this band does not change in intensity and position in the $\mathrm{rR}$ spectra in the presence of glyphosate, suggesting that the change observed on the SERS substrate comes from re-orientation of the 2 vinyl group of the hemin molecule over the surface of the silver substrate. Figure 6B shows an increase of the band at $372 \mathrm{~cm}^{-1}$ without a change in its position when glyphosate is present in the hemin solutions. The presence of propionate modes under such conditions that are not observed in the insert of Supplementary Figure 4 implies an increase in the nearness of the propionate group to the silver surface. This is only conceived when considering the cofacial $\pi-\pi$ dimmer of hemin, where although there is no overlap between the porphyrin nuclei of the two FP-Fe(III) units, an overlap between the vinyl

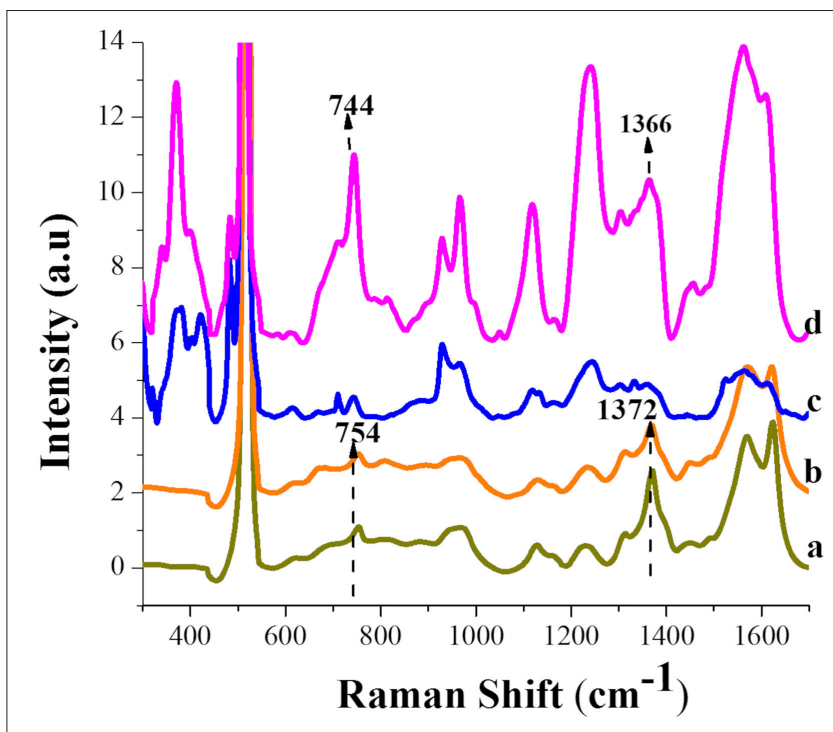

FIGURE 7 | SERS spectra acquired at $532 \mathrm{~nm}$ of excitation for: (a) $25 \mu \mathrm{M}$ of hemin-borate solution, (b) mixture of hemin-borate and $1 \mu \mathrm{M}$ glyphosate solutions. SERS spectra acquired at $780 \mathrm{~nm}$ of excitation for: (c) the same solution as in (a), (d) the same solution as in (b). Range: 540-1680 nm; incubation time: $48 \mathrm{~h}$.

group of one unit and the porphyrin group of a second unit is present (Klonis et al., 2010). Thus, an approach of the vinyl groups of the hemin dimmers to the Ag surface also implies the proximity of the propionate group.

On the other hand, as shown in Figure $6 \mathrm{~A}$ and Table 1, the enhanced modes of hemin in the presence of glyphosate at an excitation of $780 \mathrm{~nm}$ include the A1g and B1g type modes. At this wavelength in resonance Raman spectroscopy experiments on hemin solutions, the $\mathrm{A} 1 \mathrm{~g}$ and B1g type modes increase in intensity, compared with other excitation wavelengths (Franzen et al., 2002). Therefore, the observed increase in SERS, may come from a resonance Raman phenomenon: a modification in the electronic distribution of hemin as a result of a change in its heme-iron valence, corroborating that these modes arise from a charge transfer (CT) transition of heme by a vibronic coupling mechanism (Wood et al., 2004).

To contrast the results obtained at $780 \mathrm{~nm}$, SERS measurements of the same solutions were recorded using excitation with a laser of $532 \mathrm{~nm}$, where an electronic transition (Q band) and a LSPR absorption signal in the Si-AgNPs substrates also occur. Therefore, in the same way as in the case of excitation at $780 \mathrm{~nm}$, electromagnetic and chemical contributions are expected for the obtained SERS response. Figure 7 shows the SERS spectra obtained at $532 \mathrm{~nm}$ of excitation wavelength for hemin borate solution before (curve a) and after (curve b) $48 \mathrm{~h}$ of incubation with $10 \mathrm{mM}$ glyphosate solutions. For comparison, the respective SERS spectra obtained with excitation at $780 \mathrm{~nm}$ are added (curves $\mathrm{c}$ and $\mathrm{d}$ ).

At $532 \mathrm{~nm}$, the domain of modes attributable to hemin vinylic groups in the absence and presence of glyphosate are observed. However, the position of the $\mathrm{C}=\mathrm{C}$ mode at $1,620 \mathrm{~cm}^{-1}$ and that 

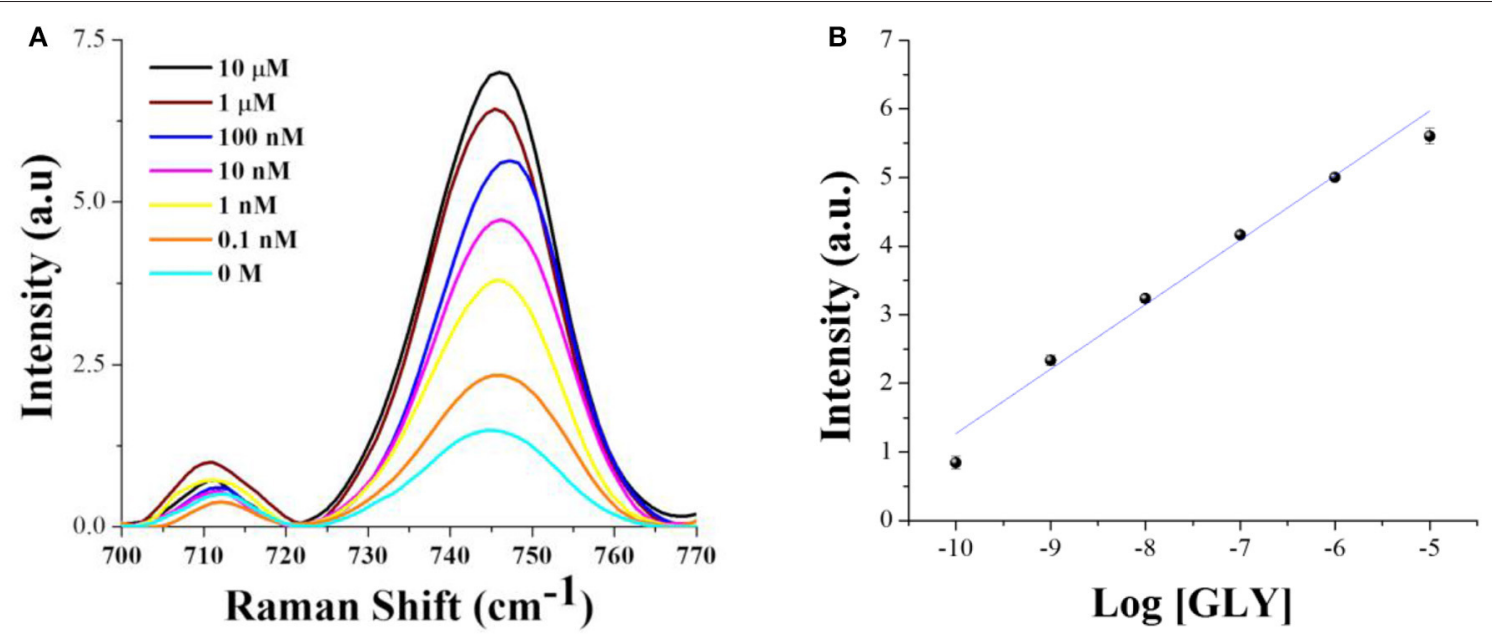

FIGURE 8 | (A) SERS spectra at $780 \mathrm{~nm}$ excitation wavelength for different glyphosate concentrations in $25 \mu \mathrm{M}$ of hemin-borate solutions after $48 \mathrm{~h}$ of incubation. (B) Calibration curve.

of the low-frequency modes associated with the bending modes of the vinyl (from 320 to $456 \mathrm{~cm}^{-1}$ ) do not show changes in the presence of glyphosate. This behavior allows inferring that hemin is adsorbed through its vinyl groups, but does not undergo a planar orientation change on silver substrates. The $v 4$ mode at $1,372 \mathrm{~cm}^{-1}$ indicates the ferric state, Fe(III) (DeVito and Asher, 1989). This information is confirmed by the presence of the $\nu 15$ mode at $754 \mathrm{~cm}^{-1}$, as considered by Zheng et al. (2003). Thus, from the observed at both 780 and $532 \mathrm{~nm}$ excitations, a change in the conformation of the vinyl groups on silver can provoke the affectation of the heme iron redox potential, as it has been inferred in the literature (Chen et al., 2004). On the other hand, it is corroborated that the $\nu 15$ mode is also sensitive to the redox state of the heme iron.

\section{Glyphosate Quantification}

Indirect quantification of glyphosate by SERS at $780 \mathrm{~nm}$ excitation wavelength was achieved by monitoring the pyridine ring breathing mode of hemin at $745 \mathrm{~cm}^{-1}$ with the contribution of the negatively charged borate ion $\left(\mathrm{B}(\mathrm{OH})_{4}^{-}\right)$, which increases with the herbicide concentration. It is important to highlight that in the absence of hemin at the same experimental conditions, this band does not show a clear intensity tendency to allow quantification (Supplementary Figure 5). Figure 8A corresponds to the SERS spectra of hemin solutions with different glyphosate concentrations after $48 \mathrm{~h}$ of incubation. All the spectra were normalized against the band at $715 \mathrm{~cm}^{-1}$, and the intensity values were settled to zero at the foot of the band $\left(720 \mathrm{~cm}^{-1}\right)$. Figure 8B shows the calibration curve constructed by plotting the intensity of the bands at $745 \mathrm{~cm}^{-1}$ vs. $\log [\mathrm{GLY}]$. The intensity values were corrected by subtracting the intensity of the curve obtained in the absence of glyphosate.

A determination coefficient $R^{2}$ of 0.9801 was obtained from the fitted curve, with excellent experimental repeatability as observed from the error bars that comprise the standard deviations from three independent measurements. The equation describing this relationship is:

Intensity $=0.9403 \log [\mathrm{GLY}]+10.6718$

The linear range is from $1 \times 10^{-10}$ to $1 \times 10^{-5} \mathrm{M}$. The limit of detection (LOD) was $9.59 \times 10^{-12} \mathrm{M}$ (obtained from 3 standard deviations from the average of the blank sample). This value is lower than that found with the Abraxis method $\left(8.87 \times 10^{-8} \mathrm{M}\right)$, which is considered one of the most flexible and versatile enzymelinked immunosorbent assay (ELISA) to detect glyphosate, and the accuracy is compared to standard liquid chromatography and tandem mass spectrometry methods (Berg et al., 2018). Table 2 shows a comparison of the LOD obtained with different methodologies, including SERS. As it can be noted, the LOD in this work is three and two orders of magnitude higher than in our previously published electrochemical method and the one reported by Cao et al. (2019), respectively, but lower than the other shown methods. Also, the limit of quantification (LOQ) attained in this work was $5.69 \times 10^{-11} \mathrm{M}$.

\section{Glyphosate Quantification in Honey}

As an example of the applicability of the quantification proposal, organic honey samples collected from a local market were spiked with different concentrations of glyphosate $\left(1 \times 10^{-9}, 1 \times 10^{-8}\right.$, $1 \times 10^{-7}, 1 \times 10^{-6}$, and $\left.1 \times 10^{-5} \mathrm{M}\right)$. Before the hemin incubation step $(48 \mathrm{~h})$, which precedes SERS measurements, the $\mathrm{pH}$ of honey samples dissolved in borate solution was adjusted to 9.2 with $\mathrm{NaOH}$ in order to obtain a homogeneous mixture with hemin. The accuracy and precision of the method were tested via recovery experiments. Figure 9 shows the SERS response of the incubated solutions of honey/hemin in the absence and the presence of different concentrations of glyphosate.

From Figure 9, it is clear that no Raman signal comes from honey or sodium hydroxide in the range from 700 to $770 \mathrm{~cm}^{-1}$. Quantification of glyphosate was conducted using the band at $715 \mathrm{~cm}^{-1}$ for normalization, and the band at $745 \mathrm{~cm}^{-1}$ was employed as the marker band. Table 3 shows the recovery values 
TABLE 2 | LOD values for glyphosate detection from different methodologies.

\begin{tabular}{|c|c|c|c|}
\hline Technique & LOD (mol L-1) & System/condition & References \\
\hline Electrochemistry & $1.4 \times 10^{-13}$ & $\begin{array}{l}\text { ITO electrode modified with copper and } \\
\text { benzene-1,3,5-tricarboxylic acid }\end{array}$ & Cao et al., 2019 \\
\hline Electrochemistry & $7.92 \times 10^{-15}$ & $\begin{array}{l}\text { Glassy carbon modified with multiwalled } \\
\text { carbon nanotubes and horse-radish peroxidase }\end{array}$ & $\begin{array}{l}\text { Cahuantzi-Muñoz } \\
\text { et al., } 2019\end{array}$ \\
\hline UV-Vis spectroscopy & $5.32 \times 10^{-6}$ & Citrate coated silver nanoparticles (colloidal) & De Góes et al., 2017 \\
\hline SERS & $1.89 \times 10^{-5}$ & & \\
\hline $\begin{array}{l}\text { Sequential-injection } \\
\text { reversed-phase } \\
\text { chromatography }\end{array}$ & $3 \times 10^{-8}$ & Pre-column derivatization & $\begin{array}{l}\text { Oliveira Pereira } \\
\text { et al., } 2019\end{array}$ \\
\hline SERS & $1.43 \times 10^{-8}$ & $\begin{array}{l}\text { GLY derivatization with ninhydrin/colloidal silver } \\
\text { nanoparticles }\end{array}$ & Xu et al., 2018 \\
\hline SERS & $9.59 \times 10^{-12} \mathrm{M}$ & $\begin{array}{l}\text { Si-AgNPs substrate/use of hemin as a reporter } \\
\text { molecule }\end{array}$ & This work \\
\hline
\end{tabular}

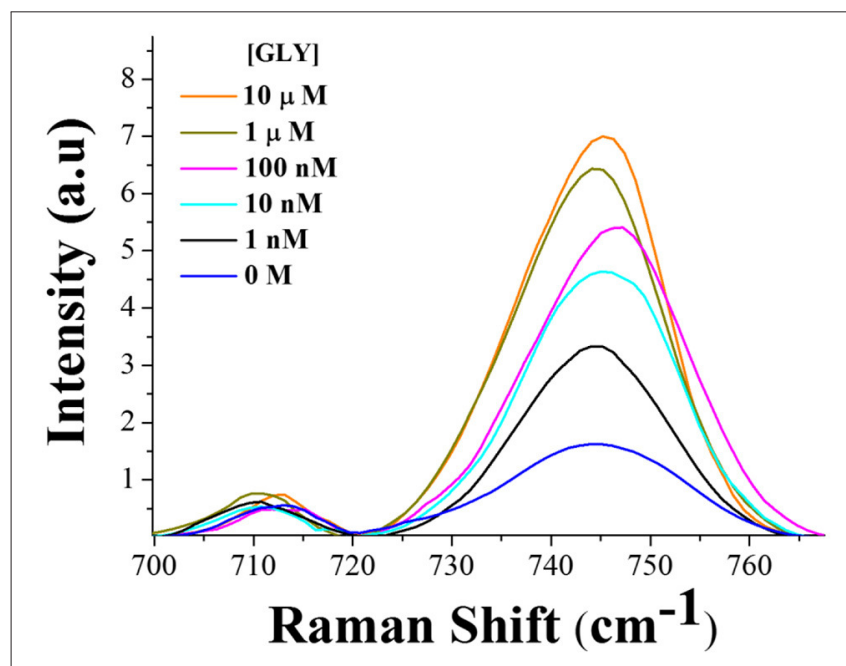

FIGURE 9 | SERS spectra taken at the excitation wavelength of $780 \mathrm{~nm}$ for the mixture of hemine $25 \mu \mathrm{M}+$ borate solution $0.25 \mathrm{M}$, after $48 \mathrm{~h}$ of incubation with different concentrations of glyphosate $(0-10 \mu \mathrm{M})$ spiked in honey samples.

TABLE 3 | Accuracy and precision obtained with the SERS sensing system.

\begin{tabular}{lcc}
\hline Added [GLY] $\left(\mathbf{m o l ~ L}^{-\mathbf{1}}\right)$ & Calculated [GLY] $\left(\mathrm{mol} \mathrm{L}^{-\mathbf{1}}\right)$ & \% Recovery \\
\hline $1 \times 10^{-9}$ & $0.866 \times 10^{-9}$ & 135.20 \\
$1 \times 10^{-8}$ & $0.958 \times 10^{-8}$ & 122.69 \\
$1 \times 10^{-7}$ & $1.138 \times 10^{-7}$ & 118.96 \\
$1 \times 10^{-6}$ & $1.061 \times 10^{-6}$ & 92.29 \\
$1 \times 10^{-5}$ & $0.526 \times 10^{-5}$ & 40.13 \\
\hline
\end{tabular}

that are related to accuracy. Recovery values were between 92 and $135 \%$ for concentrations from $1 \times 10^{-9}$ to $1 \times 10^{-6} \mathrm{M}$, which is indicative of the absence of matrix effects. A lower recovery (40\%) was obtained for the higher concentration surveyed (1 $\left.\times 10^{-5} \mathrm{M}\right)$. This fact is not surprising since it is known that
SERS measurements can have deviations at high concentrations (Sackmann and Materny, 2006).

The maximum residue limit (MRL) for glyphosate in honey is established at $50 \mu \mathrm{g} \mathrm{kg}^{-1}$ by the European Union (E. U., 2013). In this work, the linear range is $0.116-1,165 \mu \mathrm{g} \mathrm{kg}^{-1}$ for honey samples, and the EU MRL value is within this interval. The limit of quantification (LOQ) obtained from 10 times the standard deviation of the blank sample average was $11.6 \mathrm{ng} \mathrm{kg}{ }^{-1}$, representing values far below the EU MRL. It is important to mention that the scope of this work is to present a concept of glyphosate sensing based on SERS measurements with the use of a reporter molecule (hemin) that can be promising as an alternative methodology. Further studies will include the influence of possible interferents such as glufosinate, aminomethylphosphonic acid (AMPA), and other herbicides.

\section{CONCLUSIONS}

In this work, we report a SERS approach for indirect quantification using a reporter molecule (RM) under a previous incubation process with the analyte, a method that has not been reported previously. The key concept is based on implementing an incubation step that allows a binding equilibrium process between the RM and the analyte, since we demonstrated that this step may influence the Raman signal reproducibility, which is an important aspect pursued in this research area. Additionally, because the incubation process is not conducted directly on the SERS substrate, oxidation or dissolution of metallic nanoparticles are prevented. Using this proposal, the quantification of glyphosate at $780 \mathrm{~nm}$ of laser excitation wavelength was possible by following the changes in a band that belongs to the hemin used as the RM, resulting in a LOD value as low as $1 \times 10^{-13}$ M. It was also successfully tested in real honey samples without the interference of the sample matrix. On the other hand, it was found that in the presence or absence of glyphosate, hemin is the adsorbed species on SERS substrates through its vinyl groups and undergoes the reduction of its prosthetic group. On the other hand, comparing the results with those using $532 \mathrm{~nm}$, it was 
corroborated that the position of the $\nu 15$ mode of hemin can be used together with the well-known oxidation-state marker band ( $v 4$ mode) to determine the redox state of heme iron. Finally, the method opens the possibility of exploring the other RManalyte systems where the binding equilibrium occurs during the incubation process.

\section{DATA AVAILABILITY STATEMENT}

The original contributions presented in the study are included in the article/Supplementary Material, further inquiries can be directed to the corresponding authors.

\section{AUTHOR CONTRIBUTIONS}

KL-C: conducted SERS experiments. LO-F: conducted UV-Vis experiments and helped in spectra interpretation. EM: conducted AFM measurements and supported with the data treatment and interpretation. EQ-G: elaborated SERS substrates, made reflectance spectroscopy, treated, and discussed data. MG-F: interpreted and discussed results, planned experiments, wrote, and submitted the article. AM-A: interpreted and discussed

\section{REFERENCES}

Aarabi, M., Soorkia, S., Grégoire, G., Broquier, M., de la Lande, A., Soep, B., et al. (2019). Water binding to Fe III hemes studied in a cooled ion trap: characterization of a strong 'weak' ligand. Phys. Chem. Chem. Phys. 21:21329. doi: 10.1039/C9CP03608C

Aca-López, V., Quiroga-González, E., Gómez-Barojas, E., Swiatowska, J., and Luna-López, J. A. (2020). Effects of the doping level in the production of silicon nanowalls by metal assisted chemical etching. Mat. Sci. Semicon. Proc. 118:105206. doi: 10.1016/j.mssp.2020.105206

Alizadeh, N., Salimi, A., and Hallaj, R. (2017). "Hemin/G-Quadruplex horseradish peroxidase-mimicking DNAzyme: principle and biosensing application," in Catalytically Active Nucleic Acids, eds H. Seitz, F. Stahl, and J.-G. Walter (Cham: Springer), 85-106. doi: 10.1007/10_2017_37

Amirjani, A., and Haghshenas, D. F. (2018). Ag nanostructures as the surface plasmon resonance (SPR)-based sensors: a mechanistic study with an emphasis on heavy metallic ions detection. Sensor. Actuat. B-Chem. 273:1768. doi: 10.1016/j.snb.2018.07.089

Aratani, N., Osuka, A., Cho, H. S., and Kim, D. (2002). Photochemistry of covalently-linked multi-porphyrinic systems. Photochem. Photobiol. C Photochem. Rev. 3:25. doi: 10.1016/S1389-5567(02)00003-5

Arbelo-López, H. D., Rodriguez-Mackenzie, A. D., Roman-Morales, E. M., Wymore, T., and López-Garriga, J. (2018). Charge transfer and $\pi$ to $\pi^{*}$ transitions in the visible spectra of sulfheme met isomeric structures. J. Phys. Chem. B 122:4947. doi: 10.1021/acs.jpcb.7b12393

Avino, P., Notardonato, I., and Russo, M. V. (2020). "A review of the analytical methods based on chromatography for analyzing glyphosate in foods," in Pests, Weeds and Diseases in Agricultural Crop and Animal Husbandry Production, eds D. Kontogiannatos, A. Kourti, and K. F. Mendes (London: IntechOpen), 1-24. doi: 10.5772/intechopen.92810

Berg, C. J., King, H. P., Delenstarr, G., Kumar, R., Rubio, F., and Glaze, T. (2018). Glyphosate residue concentrations in honey attributed through geospatial analysis to proximity of large-scale agriculture and transfer off-site by bees. PLoS ONE 13:e0198876. doi: 10.1371/journal.pone.01 98876

Cahuantzi-Muñoz, S., González-Fuentes, M., Ortiz-Frade, L., Torres, E., Țălu, S., Trejo, G., et al. (2019). Electrochemical Biosensor for sensitive quantification of glyphosate in maize kernels. Electroanal. 31:927. doi: 10.1002/elan.2018 00759 results, planned experiments, and wrote the article. All authors contributed to the article and approved the submitted version.

\section{FUNDING}

KL-C was grateful to the Consejo Nacional de Ciencia y Tecnología (CONACYT) for her doctorate fellowship. LO-F was grateful for financial support from CONACyT through the projects Apoyo a la infraestructura 2016(269102) and CONACyT-SEP Ciencia Básica (288069). EM, MG-F, and AM-A acknowledged BUAP for its support through VIEP projects.

\section{ACKNOWLEDGMENTS}

The authors thank Dr. Andrey Kravey from HORIBA Scientific for his help with AFM analysis.

\section{SUPPLEMENTARY MATERIAL}

The Supplementary Material for this article can be found online at: https://www.frontiersin.org/articles/10.3389/fchem. 2020.612076/full\#supplementary-material

Cao, Y., Wang, L., Wang, C., Su, D., Liu, Y., and Hu, X. (2019). An electrochemical sensor on the hierarchically porous Cu-BTC MOF platform for glyphosate determination. Sensor Actuat. B 283:487. doi: 10.1016/j.snb.2018.12.064

Chen, S., Ding, C., Lin, Y., Wu, X., Yuan, W., Meng, X., et al. (2020). SERS-active substrate assembled by Ag NW-embedded porous polystyrene fibers. RSC Adv. 10:21845. doi: 10.1039/D0RA01454K

Chen, Z., Ost, T. W., and Schelvis, J. P. (2004). Phe393 mutants of cytochrome P450 BM3 with modified heme redox potentials have altered heme vinyl and propionate conformations. Biochem. 43:1798. doi: 10.1021/bi034920g

Choi, S., Spiro, T., Langry, K., and Smith, K. (1982). Vinyl influences on protoheme resonance Raman spectra: nickel (II) protoporphyrin IX with deuterated vinyl groups. J. Am. Chem. Soc. 104:4337. doi: 10.1021/ja00380a005

Cui, L., Wu, D. Y., Wang, A., Ren, B., and Tian, Z. Q. (2010). Chargetransfer enhancement involved in the SERS of adenine on $\mathrm{Rh}$ and $\mathrm{Pd}$ demonstrated by ultraviolet to visible laser excitation. J. Phys. Chem. C 114:16588. doi: 10.1021/jp1055717

De Góes, R. E., Muller, M., and Fabris, J. L. (2017). Spectroscopic detection of glyphosate in water assisted by laser-ablated silver nanoparticles. Sensors 17:954. doi: 10.3390/s17050954

Demirel, G., Usta, H., Yilmaz, M., Celik, M., Alidagi, H. A., and Buyukserin, F. (2018). Surface-enhanced Raman spectroscopy (SERS): an adventure from plasmonic metals to organic semiconductors as SERS platforms. J. Mater. Chem. C 6:5314. doi: 10.1039/C8TC01168K

Desbois, A., Henry, Y., and Lutz, M. (1984). Influence of peripheral substituents on the resonance Raman spectra of ferroporphyrin-2-methylimidazole complexes. BBA - Protein. Struct. M. 785, 148-160. doi: 10.1016/0167-4838(84)90139-0

DeVito, V. L., and Asher, S. A. (1989). UV resonance Raman enhancement of vinyl stretching in ferric protoporphyrin IX: conjugation and preservation of the vinyl $\pi \rightarrow \pi^{*}$ transition. J. Am. Chem. Soc. 111:9143. doi: 10.1021/ja00208a001

Drozdowicz-Tomsia, K., and Goldys, E. M. (2011). "Gold and silver nanowires for fluorescence enhancement," in Nanowires Fundamental Research, ed A. Hashim (London: IntechOpen), 309-332.

Ehrl, B. N., Mogusu, E. O., Kim, K., Hofstetter, H., Pedersen, J. A., and Elsner, M. (2018). High permeation rates in liposome systems explain rapid glyphosate biodegradation associated with strong isotope fractionation. Environ. Sci. Technol. 52:7259. doi: 10.1021/acs.est.8b01004

E. U. (2013). Commission Regulation (EU) No 293/2013 of 20 March 2013 amending Annexes II and III to Regulation (EC) No 396/2005 of the European Parliament and of the Council as regards maximum residue levels 
for emamectin benzoate, etofenprox, etoxazole, flutriafol, glyphosate, phosmet, pyraclostrobin, spinosad and spirotetramat in or on certain products. Off. J. Eur. Union 96, 1-30.

Feis, A., Gellini, C., Ricci, M., Tognaccini, L., Becucci, M., and Smulevich, G. (2020). Surface-enhanced Raman scattering of glyphosate on dispersed silver nanoparticles: a reinterpretation based on model molecules. Vib. Spectrosc. 108:103061. doi: 10.1016/j.vibspec.2020.103061

Franzen, S., Wallace-Williams, S. E., and Shreve, A. P. (2002). Heme chargetransfer band III is vibronically coupled to the Soret band. J. Am. Chem. Soc. 124:7146. doi: $10.1021 / \mathrm{ja} 0172722$

He, J., Yang, H., Zhang, Y., Yu, J., Miao, L., Song, Y., et al. (2016). Smart nanocomposites of $\mathrm{Cu}$-hemin metal-organic frameworks for electrochemical glucose biosensing. Sci. Rep. 6:36637. doi: 10.1038/srep36637

Hu, S., Smith, K. M., and Spiro, T. G. (1996). Assignment of protoheme resonance Raman spectrum by heme labeling in myoglobin. J. Am. Chem. Soc. 118, 12638-12646. doi: 10.1021/ja962239e

IARC (2017). Some Organophosphate Insecticides and Herbicides. Lyon (FR): International Agency for Research on Cancer, 398.

Klonis, N., Dilanian, R., Hanssen, E., Darmanin, C., Streltsov, V., Deed, S., et al. (2010). Hematin- hematin self-association states involved in the formation and reactivity of the malaria parasite pigment, hemozoin. Biochemistry 49:6804. doi: 10.1021/bi100567j

Kosović, M., Balarin, M., Ivanda, M., Đerek, V., Marciuš, M., Ristić, M., et al. (2015). Porous silicon covered with silver nanoparticles as surface-enhanced Raman scattering (SERS) substrate for ultra-low concentration detection. Appl. Spectrosc. 69:1417. doi: 10.1366/14-07729

Lee, J., Min, K., Kim, Y., and Yu, H. K. (2019). Surface-enhanced raman spectroscopy (SERS) study using oblique angle deposition of Ag using different substrates. Materials 12:1581. doi: 10.3390/ma12101581

Lee, K. C., Lin, S. J., Lin, C. H., Tsai, C. S., and Lu, Y. J. (2008). Size effect of Ag nanoparticles on surface plasmon resonance. Surf. Coat. Tech. 202, 5339-5342. doi: 10.1016/j.surfcoat.2008.06.080

Li, L., Liu, C., Cao, X., Tan, L., and Lu, W. (2017). Multiplexing determination of cancer-associated biomarkers by surface-enhanced Raman scattering using ordered gold nanohoneycomb arrays. Bioanalysis 9:1561. doi: 10.4155/bio-2016-0237

Li, M., Qiu, Y., Fan, C., Cui, K., Zhang, Y., and Xiao, Z. (2018). Design of SERS nanoprobes for Raman imaging: materials, critical factors and architectures. Acta. Pharm. Sinica. B 8:381. doi: 10.1016/j.apsb.2018.01.010

Lin, T., Song, Y., Liao, J., Liu, F., and Zeng, T. (2020). Applications of surfaceenhanced Raman spectroscopy in detection fields. Nanomedicine [Preprint]. Available online at: https://www.futuremedicine.com/doi/full/10.2217/nnm2020-0361 (accessed November 15, 2020).

Liu, S., Hu, C., Wei, Y., Duan, M., Chen, X., and Hu, Y. (2018). Transformation of $\mathrm{H}$-aggregates and J-dimers of water-soluble tetrakis (4carboxyphenyl) porphyrin in polyion complex micelles. Polymers 10:494. doi: $10.3390 /$ polym 10050494

Lopes Catão, A. J., and López-Castillo, A. (2018). On the degradation pathway of glyphosate and glycine. Environ. Sci. Processes 20:1148. doi: 10.1039/C8EM00119G

Lu, S., You, T., Yang, N., Gao, Y., and Yin, P. (2020). Flexible SERS substrate based on Ag nanodendrite-coated carbon fiber cloth: simultaneous detection for multiple pesticides in liquid droplet. Anal. Bioanal. Chem. 412:1159. doi: 10.1007/s00216-019-02344-6

Maehly, A. C., and Akeson, A. (1958). Stabilization of aqueous hemin solutions. Acta. Chem. Scand. 12:1259. doi: 10.3891/acta.chem.scand.12-1259

Mao, P., Liu, C., Chen, Q., Han, M., Maier, S. A., and Zhang, S. (2020). Broadband SERS detection with disordered plasmonic hybrid aggregates. Nanoscale 12:93. doi: 10.1039/C9NR08118F

März, A., Mönch, B., Walter, A., Bocklitz, T., Schuhmacher, W., Rösch, P., et al. (2011). "The multifunctional application of microfluidic lab-on-a-chip surface enhanced Raman spectroscopy (LOC-SERS) within the field of bioanalytics," in Clinical and Biomedical Spectroscopy and Imaging II (Optical Society of America), 808707 (Munich). doi: 10.1364/ECBO.2011.808707

Marzocchi, M. P., and Smulevich, G. (2003). Relationship between heme vinyl conformation and the protein matrix in peroxidases. J. Raman Spectrosc. 34:725. doi: $10.1002 /$ jrs. 1037
Milazzo, L., Exertier, C., Becucci, M., Freda, I., Montemiglio, L. C., Savino, C., et al. (2020). Lack of orientation selectivity of the heme insertion in murine neuroglobin revealed by resonance Raman spectroscopy. FEBS J. 287:4082. doi: $10.1111 /$ febs.15241

Mosier-Boss, P. A. (2017). Review of SERS substrates for chemical sensing. Nanomaterials 7:142. doi: 10.3390/nano7060142

Murgida, D. H., and Hildebrandt, P. (2001). Heterogeneous electron transfer of cytochrome $\mathrm{c}$ on coated silver electrodes. Electric field effects on structure and redox potential. J. Phys. Chem. B 105:1578. doi: 10.1021/jp003742n

Nath, A., Dharmadhikari, J., Dharmadhikari, A., Mathur, D., and Mazumdar, S. (2017). Ultrafast dynamics of hemin aggregates. Phys. Chem. Chem. Phys. 19:26862. doi: 10.1039/C7CP04858K

Norrel, J., deCastro, M., Petit, L., Cardinal, T., Guillen, F., Richardson, K. A., et al. (2003). "Anion exchange of Oxygen by Sulfur in GeO2-based glasses," in Proc. SPIE 4990, Rare-Earth-Doped Materials and Devices VII. 4990 (San Jose, CA), 87. doi: $10.1117 / 12.501140$

Oliveira Pereira, E. A., Freitas Melo, V., Abate, G., and Masini, J. C. (2019). Determination of glyphosate and aminomethylphosphonic acid by sequential-injection reversed-phase chromatography: method improvements and application in adsorption studies. Anal. Bioanal. Chem. 411:2317. doi: 10.1007/s00216-019-01672-x

Pérez-Jiménez, A. I., Lyu, D., Lu, Z., Liu, G., and Ren, B. (2020). Surface-enhanced Raman spectroscopy: benefits, trade-offs and future developments. Chem. Sci. 11:4563. doi: 10.1039/D0SC00809E

Peters, R. F., Gutierrez-Rivera, L., Dew, S. K., and Stepanova, M. (2015). Surface enhanced Raman spectroscopy detection of biomolecules using EBL fabricated nanostructured substrates. Jove-j. Vis. Exp. 2015:e52712. doi: 10.3791/52712

Pilot, R., Signorini, R., Durante, C., Orian, L., Bhamidipati, M., and Fabris, L. (2019). A review on surface-enhanced raman scattering. Biosensors 9:57. doi: 10.3390/bios 9020057

Pyrak, E., Krajczewski, J., Kowalik, A., Kudelski, A., and Jaworska, A. (2019). Surface enhanced raman spectroscopy for DNA biosensors-how far are we? Molecules 24:4423. doi: 10.3390/molecules 24244423

Rwere, F., Mak, P. J., and Kincaid, J. R. (2008). Resonance Raman interrogation of the consequences of heme rotational disorder in myoglobin and its ligated derivatives. Biochemistry 47:12869. doi: 10.1021/bi801779d

Sackmann, M., and Materny, A. (2006). Surface enhanced Raman scattering (SERS)-a quantitative analytical tool? J. Raman Spectrosc. 37:305. doi: $10.1002 /$ jrs. 1443

Sánchez-Purrà, M., Roig-Solvas, B., Rodriguez-Quijada, C., Leonardo, B. M., and Hamad-Schifferli, K. (2018). Reporter selection for nanotags in multiplexed surface enhanced raman spectroscopy assays. ACS omega 3:10733. doi: 10.1021/acsomega.8b01499

Scolaro, L. M., Castriciano, M., Romeo, A., Patanè, S., Cefalì, E., and Allegrini, M. (2002). Aggregation behavior of protoporphyrin IX in aqueous solutions: clear evidence of vesicle formation. J. Phys. Chem. B 106:2453. doi: $10.1021 / \mathrm{jp} 013155 \mathrm{~h}$

Sharma, V., Verma, D., and Okram, G. S. (2020). Influence of surfactant, particle size and dispersion medium on surface plasmon resonance of silver nanoparticles. J. Phys-Condens. Mat. 32:145302. doi: 10.1088/1361-648X/ab601a

Steinborn, A., Alder, L., Michalski, B., Zomer, P., Bendig, P., Martinez, S. A., et al. (2016). Determination of glyphosate levels in breast milk samples from Germany by LC-MS/MS and GC-MS/MS. J. Agr. Food. Chem. 64:1414. doi: $10.1021 /$ acs.jafc.5b05852

Tan, M. J., Hong, Z.-Y., Chang, M.-H., Liu, C.-C., Cheng, H.-F., Loh, X. J., et al. (2017). Metal carbonyl-gold nanoparticle conjugates for highly sensitive SERS detection of organophosphorus pesticides. Biosens. Bioelectron. 96:167. doi: 10.1016/j.bios.2017.05.005

Toader, A. M., Ţome, M., and Volanschi, E. (2013). Hemin in vitro interaction with the anticancer drug doxorubicin: absorption and emission monitoring. Rev. Roum. Chim. 58, 415-423.

Torul, H., Boyaci, İ. H., and Tamer, U. (2010). Attomole detection of glyphosate by surface-enhanced Raman spectroscopy using gold nanorods. J. Pharm. Sci. $35,179-184$.

Turner, J. A. (2020). The Pesticide Manual: A World Compendium. Aldershot: British Crop Protection Council. 
Webster, G. T., McNaughton, D., and Wood, B. R. (2009). Aggregated enhanced Raman scattering in Fe (III) PPIX solutions: the effects of concentration and chloroquine on excitonic interactions. J. Phys. Chem. B 113:6910. doi: $10.1021 /$ jp811028a

Wood, B. R., Bailo, E., Khiavi, M. A., Tilley, L., Deed, S., Deckert-Gaudig, T., et al. (2011). Tip-enhanced Raman scattering (TERS) from hemozoin crystals within a sectioned erythrocyte. Nano lett. 11, 1868-1873. doi: 10.1021/nl103004n

Wood, B. R., Langford, S. J., Cooke, B. M., Lim, J., Glenister, F. K., Duriska, M., et al. (2004). Resonance Raman spectroscopy reveals new insight into the electronic structure of $\beta$-hematin and malaria pigment. J. Am. Chem. Soc. 126:9233. doi: $10.1021 / \mathrm{ja} 038691 \mathrm{x}$

Wood, B. R., Tait, B., and McNaughton, D. (2001). Micro-Raman characterization of the $\mathrm{R}$ to $\mathrm{T}$ state transition of haemoglobin within a single living erythrocyte. Bba-Mol. Cell. Res. 1539:58. doi: 10.1016/S0167-4889(01)00 089-1

Xu, M.-L., Gao, Y., Li, Y., Li, X., Zhang, H., Han, X. X., et al. (2018). Indirect glyphosate detection based on ninhydrin reaction and surfaceenhanced Raman scattering spectroscopy. Spectrochim. Acta A 197:78. doi: 10.1016/j.saa.2018.01.014

Yin, Z., He, H., Wang, Z., Fang, X., Xu, C., Luo, D., et al. (2020). Facile in situ photochemical synthesis of silver nanoaggregates for surface-enhanced raman scattering applications. Nanomaterials 10:685. doi: 10.3390/nano10040685

Yongquan, Z., Chunhui, F., Yan, F., and Fayan, Z. (2013). Density, electrical conductivity, acidity, viscosity and Raman spectra of aqueous $\mathrm{NaBO}_{2}, \mathrm{Na}_{2} \mathrm{~B}_{4} \mathrm{O}_{7}$ and $\mathrm{NaB}_{5} \mathrm{O}_{8}$ solutions at 298.15 and $323.15 \mathrm{~K}$. J. Chem. Soc. Pak. 35, 166-1071.
Zhang, T., Wang, L., Gao, C., Zhao, C., Wang, Y., and Wang, J. (2018). Hemin immobilized into metal-organic frameworks as electrochemical biosensor for 2,4,6-trichlorophenol. Nanotechnology 29:074003. doi: 10.1088/1361-6528/a aa26e

Zhao, X., Li, M., and Xu, Z. (2018). Detection of foodborne pathogens by surface enhanced Raman spectroscopy. Front. Microbiol. 9:1236. doi: $10.3389 /$ fmicb.2018.01236

Zheng, J. W., Li, X. W., Xu, H. Y., Zhou, Y. G., and Gu, R. A. (2003). A non-resonance surface-enhanced Raman spectroscopic study of hemin on a roughened silver electrode. Guang pu xue yu guang pu fen xi 23, 294-296.

Zou, H. L., Li, B. L., Luo, H. Q., and Li, N. B. (2015). A novel electrochemical biosensor based on hemin functionalized graphene oxide sheets for simultaneous determination of ascorbic acid, dopamine and uric acid. Sensor. Actuat. B Chem. 207:535. doi: 10.1016/j.snb.2014.10.121

Conflict of Interest: The authors declare that the research was conducted in the absence of any commercial or financial relationships that could be construed as a potential conflict of interest.

Copyright (c) 2020 López-Castaños, Ortiz-Frade, Méndez, Quiroga-González, González-Fuentes and Méndez-Albores. This is an open-access article distributed under the terms of the Creative Commons Attribution License (CC BY). The use, distribution or reproduction in other forums is permitted, provided the original author(s) and the copyright owner(s) are credited and that the original publication in this journal is cited, in accordance with accepted academic practice. No use distribution or reproduction is permitted which does not comply with these terms. 